\title{
Mild hypoglycemia is independently associated with increased mortality in the critically ill
}

\author{
James S Krinsley ${ }^{1 *}$, Marcus J Schultz ${ }^{2,3}$, Peter E Spronk ${ }^{2,4}$, Robin E Harmsen ${ }^{2}$, Floris van Braam Houckgeest ${ }^{5}$, \\ Johannes $\mathrm{P}$ van der Sluijs ${ }^{6}$, Christian Mélot $^{7}$ and Jean Charles Preiser ${ }^{8}$
}

\begin{abstract}
Introduction: Severe hypoglycemia (blood glucose concentration $(B G)<40 \mathrm{mg} / \mathrm{dL}$ ) is independently associated with an increased risk of mortality in critically ill patients. The association of milder hypoglycemia ( $\mathrm{BG}<70 \mathrm{mg} / \mathrm{dL}$ ) with mortality is less clear.

Methods: Prospectively collected data from two observational cohorts in the USA and in The Netherlands, and from the prospective GLUCONTROL trial were analyzed. Hospital mortality was the primary endpoint.

Results: We analyzed data from 6,240 patients: 3,263 admitted to Stamford Hospital (ST), 2,063 admitted to three institutions in The Netherlands (NL) and 914 who participated in the GLUCONTROL trial (GL). The percentage of patients with hypoglycemia varied from $18 \%$ to $65 \%$ among the different cohorts. Patients with hypoglycemia experienced higher mortality than did those without hypoglycemia even after stratification by severity of illness, diagnostic category, diabetic status, mean BG during intensive care unit (ICU) admission and coefficient of variation (CV) as a reflection of glycemic variability. The relative risk (RR, 95\% confidence interval) of mortality associated with minimum BG < 40, 40 to 54 and 55 to $69 \mathrm{mg} / \mathrm{dL}$ compared to patients with minimum BG 80 to $109 \mathrm{mg} / \mathrm{dL}$ was 3.55 (3.02 to 4.17), 2.70 (2.31 to 3.14) and 2.18 (1.87 to 2.53), respectively (all $P<0.0001$ ). The RR of mortality associated with any hypoglycemia $<70 \mathrm{mg} / \mathrm{dL}$ was 3.28 (2.78 to 3.87$)(P<0.0001), 1.30(1.12$ to 1.50$)(P=0.0005)$ and 2.11 (1.62 to 2.74) $(P<0.0001)$ for the ST, NL and GL cohorts, respectively. Multivariate regression analysis demonstrated that minimum BG $<70 \mathrm{mg} / \mathrm{dL}$, 40 to $69 \mathrm{mg} / \mathrm{dL}$ and $<40 \mathrm{mg} / \mathrm{dL}$ were independently associated with increased risk of mortality for the entire cohort of 6,240 patients (odds ratio (OR) (95\% confidence interval (Cl)) 1.78 (1.39 to 2.27) $P<0.0001), 1.29$ (1.11 to 1.51$) P=0.0011$ and $1.87(1.46$ to 2.40$) P<0.0001$ ) respectively.

Conclusions: Mild hypoglycemia was associated with a significantly increased risk of mortality in an international cohort of critically ill patients. Efforts to reduce the occurrence of hypoglycemia in critically ill patients may reduce mortality
\end{abstract}

\section{Introduction}

Hyperglycemia occurs commonly in critically ill patients and is strongly associated with increased risk of mortality [1-3]. Interventional trials designed to treat even moderate degrees of hyperglycemia with insulin have met with mixed success. A landmark single-center trial among a cohort of surgical intensive care unit (ICU) patients resulted in substantial reductions in mortality and morbidity in the treated patients [4]. Three years later a large observational study in a heterogeneous

\footnotetext{
* Correspondence: james.krinsley@gmail.com

'Division of Critical Care, Stamford Hospital, Columbia University College of Physicians and Surgeons, 190 West Broad Street, Stamford, CT 06902, USA Full list of author information is available at the end of the article
}

population of ICU patients reached a similar conclusion [5]. However, in the second Leuven trial of intensive insulin therapy (IIT), conducted in a cohort of medical patients, patients in the interventional arm did not demonstrate reduced mortality, but did have shorter ICU length of stay and duration of mechanical ventilation [6]. Subsequent trials of IIT were stopped prematurely due to high rates of hypoglycemia and protocol violations $[7,8]$ or demonstrated higher mortality among the intensively treated cohort [9]. In fact, the second Leuven trial was the first investigation to identify an association between severe hypoglycemia (BG $<40 \mathrm{mg} /$ dL) and mortality [6].

\section{C) Biomed Central}


A relatively low rate of severe hypoglycemia, defined as blood glucose concentration (BG) $<40 \mathrm{mg} / \mathrm{dL}$ and identified as having an independent association with mortality in several large observational cohorts [10-12], was a key feature that distinguished the first two investigations [4,5] from those that followed [6-9]. The Leuven investigators subsequently pooled data from their surgical [4] and medical [6] trials and used multivariate analysis to confirm that severe hypoglycemia had a strong and independent association with mortality [13]. A recent large observational cohort study suggested that even mild levels of hypoglycemia, defined as BG $<72$ $\mathrm{mg} / \mathrm{dL}$, were associated with increased risk of death among critically ill patients [14]. Consistently, the recently revised standards of medical care in diabetes used a threshold BG of $70 \mathrm{mg} / \mathrm{dl}$ to define hypoglycemia [15].

The purpose of this study was to evaluate the association of hypoglycemia, defined as BG $<70 \mathrm{mg} / \mathrm{dL}$, with mortality. We hypothesized that (1) hypoglycemia (BG < $70 \mathrm{mg} / \mathrm{dL}$ ) was associated with increased risk of mortality, and (2) that a glycemic management strategy that tolerated brief periods of mild hypoglycemia and corrected these events without creating marked glycemic excursions would demonstrate a weaker association with mortality than would protocols that avoided and treated mild hypoglycemia more vigorously. We had the unique opportunity to analyze a large and diverse group of critically ill patients in an international collaboration, including a large single-center cohort from 1 ICU in the United States of America, a multicenter cohort from 3 ICUs from The Netherlands, and 21 ICUs from Europe and Israel that participated in the GLUCONTROL trial, a multicenter randomized controlled trial of IIT [8].

\section{Materials and methods}

\section{Settings}

The adult ICU of Stamford Hospital (Stamford, CT, USA) is a 16-bed unit that treats a heterogeneous population of medical, surgical and trauma patients. Medical and surgical house staff, closely supervised by a team of intensivists, deliver care.

The adult ICUs of the three hospitals in The Netherlands (Gelre Hospital, Apeldoorn, The Netherlands; Tergooi Hospitals, Hilversum, The Netherlands; Medical Center Haaglanden, The Hague, The Netherlands) are a 10-bed, 9-bed and 18-bed unit, respectively, which treat a heterogeneous population of medical, surgical and trauma patients. A team of intensivists delivers care in a closed-format setting.

Twenty-one adult ICUs from 19 different hospitals in seven countries in Europe and Israel participated in the GLUCONTROL trial [8] The number of ICU beds of the participating units ranged from 5 to 44 (median 12).

\section{Patients}

The patient cohort in Stamford (ST) included 3,263 patients admitted to the ICU between 12 January 2007 and 30 April 2010, who had at least three BG values obtained during their ICU stay. Forty-one patients admitted during this period with a diagnosis of diabetic ketoacidosis or hyperosmolar nonketotic coma were excluded from the present analysis.

The patient cohort in The Netherlands (NL) included 2,063 patients admitted to the three ICUs between 1 January 2007 and 29 December 2009, who had at least three BG values obtained during their ICU stay: 1,098 patients, admitted between 1 January 2007 and 31 January 2008 were subjected to a "loose" IIT guideline; 965 patients admitted between 1 February 2008 and 29 December 2009 were subjected to a "strict" IIT guideline (see below for details on "loose" and "strict" IIT). Per protocol, patients admitted during this period with a diagnosis of diabetic ketoacidosis or hyperosmolar nonketotic coma were not subjected to treatment according to the guidelines.

The patient cohort in the GLUCONTROL trial (GL) included 914 patients with at least three BG values; the other 164 patients (those with fewer than three BG recorded) were evenly distributed between the intensive (GL-IIT) and intermediate glucose control groups (GLC).

\section{Glycemic control and blood glucose monitoring}

The glycemic target in Stamford during the period of the investigation was 80 to $125 \mathrm{mg} / \mathrm{dL}$. Details of the protocol have been published previously [16] (and are available as Additional file 1). Most of the BG measurements (85\%) were made using bedside glucometers (Accu-Chek Inform, Indianapolis, IN, USA) and capillary or venous blood; the remainder were performed in the central laboratory using a Siemens Advia 1800 analyzer (Siemens Corporation US, Washington, DC, USA) or in the ICU using a GEM4000 point of care analyzer (Instrument Laboratory, Lexington, MA, USA).

Patients in the NL cohort were treated with loose or strict IIT. With loose glucose control, the three participating ICUs followed the 2004 Surviving Sepsis Campaign Guidelines [17], and aimed for a BG $<150 \mathrm{mg} / \mathrm{dL}$. Insulin dose and route of administration (either intravenous or subcutaneous), and timing and type of BG measurement (either using capillary or arterial blood, at the bedside or in a central laboratory) were loosely defined in the guidelines in use. ICUs nurses practiced blood glucose control. With strict IIT, the ICUs followed an adjusted Leuven guideline with a glycemic target range of 80 to $110 \mathrm{mg} / \mathrm{dL}$ [18]; administration of insulin was intravenous at all times, and BG measurements were performed at the bedside. Notably, while the protocol 
aimed for $\mathrm{BG}>80 \mathrm{mg} / \mathrm{dL}$, the protocol allowed $\mathrm{BG}$ from 60 to $80 \mathrm{mg} / \mathrm{dL}$ if they were short in duration. In such case, insulin infusion was stopped. Small boluses of dextrose were given only in case of severe hypoglycemia. Blood glucose control required a high level of intuitive decision-making. All BG measurements were made using bedside glucometers (AccuChek Inform, Almere, The Netherlands) and arterial blood; capillary blood was never used.

In the GLUCONTROL trial, patients were randomized to IIT (target BG: 80 to $110 \mathrm{mg} / \mathrm{dl}$ ) or intermediate glucose control (target BG 140 to $180 \mathrm{mg} / \mathrm{dl}$ ), using an insulin protocol ([8], and available as Additional file 2). BG measurements were performed on arterial or central venous samples when a catheter was in place and a blood gas analyzer was used preferentially. Capillary samples and a specific glucometer (Accu to Check Inform, Roche Diagnostics, Mannheim, Germany) were allowed.

\section{Data collected}

In Stamford, data were abstracted from the ICU's comprehensive clinical database, including prospective collection of admission diagnosis, demographic information and calculation of the Acute Physiology and Chronic Health Evaluation II (APACHE II) scores. The database is linked to the hospital's clinical information system to retrieve glucose values and the patient's hospital discharge status. Diabetic status was determined prospectively based on all available clinical information for every patient.

The Dutch centers' data were abstracted from the National Intensive Care Evaluation (NICE) database, maintained by the NICE Foundation [19], including prospective collection of admission diagnosis, demographic information and calculation of the APACHE II scores. The database was linked to the hospital's clinical information system to retrieve BG.

For the GL cohort, the data were abstracted from the original database recorded by the study participants. An independent biostatistician managed the web-based central database.

\section{Statistical analysis}

Hypoglycemia was defined as BG $<70 \mathrm{mg} / \mathrm{dL}$. Severe hypoglycemia was defined as $B G<40 \mathrm{mg} / \mathrm{dL}$. Additional stratification included bands of a minimum BG of 40 to 54,55 to 69,70 to 79,80 to 109 and $\geq 110 \mathrm{mg} /$ $\mathrm{dL}$ We calculated the relative risk of mortality associated with increments of hypoglycemia compared to patients with a minimum BG of 80 to $109 \mathrm{mg} / \mathrm{dL}$. Additional analyses stratified patients by mean BG during ICU stay using increments of 80 to $110 \mathrm{mg} / \mathrm{dL}, 110$ to $140 \mathrm{mg} / \mathrm{dL}, 140$ to $180 \mathrm{mg} / \mathrm{dL}$ and $\geq 180 \mathrm{mg} / \mathrm{dL}$, as well as by severity of illness, using APACHE II scores, and by diabetic status and diagnostic category. We also calculated the coefficient of variation (CV) for each patient, defined as standard deviation of the mean BG/ mean BG. Multivariate analysis to assess the independent association of hypoglycemia with mortality included the following parameters found to be statistically significant at $P<0.10$ on univariate analysis: age, modified APACHE II score (age component deleted, in order to avoid colinearity with age in the multivariate analysis: age 45 to 54 two points; age 55 to 64 three points; age 65 to 74 five points; age $\geq 75$ six points), ICU LOS, diagnostic category on admission to the ICU (medical vs. surgical), mechanical ventilation, mean BG and CV. Diabetes was not associated with mortality on univariate analysis and, therefore, was not entered into the multivariate model.

Continuous data were presented as median (interquartile range), and compared using the Mann Whitney rank sum test; all chosen parameters were not normally distributed. Ordinal data were presented as percentages and compared using the Chi square test. Mortality was defined throughout as hospital- (that is, not ICU-) mortality. Statistical analysis was performed using the MedCalc statistical package version 10.1.1.6.0 (MedCalc software, Broekstraat 52, 9030 Mariakerke, Belgium). A $P$ - value of $<0.05$ was considered statistically significant.

The Stamford Hospital institutional review board approved this investigation.

The GLUCONTROL trial was approved by each institutional review board of the 21 participating hospitals. Patients, or their designated surrogates, participating in the trial gave informed consent.

Approval to conduct the study was obtained from the medical ethics committees of the three centers of the Academic Medical Center in The Netherlands, which waived the requirement for individual patient-level consent.

\section{Results}

\section{Cohort characteristics}

Table 1 illustrates differences in clinical characteristics, outcomes and frequently used metrics of glycemic control among patients in the three cohorts, as well as aggregated data for the entire population of 6,240 patients. The percentage of patients with hypoglycemia varied considerably, as did mean glucose level, glycemic variability and mortality. Table 2 details the different outcomes of patients with and without hypoglycemia. For the entire cohort of 6,240, patients with hypoglycemia were older, had higher APACHE II scores, ICU LOS and mortality and also had higher indices of glycemic variability and lower mean BG during ICU stay (all comparisons $P<0.0001$ ). 
Table 1 Characteristics of the patient cohorts

\begin{tabular}{|c|c|c|c|c|c|c|}
\hline & ALL & ST & NL-L & NL-S & GL-C & GL-IIT \\
\hline \multicolumn{7}{|l|}{ Demographics and outcomes } \\
\hline Number & 6,240 & 3,263 & 1,098 & 965 & 460 & 454 \\
\hline Age & 68 (54 to 78$)$ & 68 (53 to 80) & 69 (57 to 78$)$ & 68 (56 to 78$)$ & 65 (53 to 74$)$ & 65 (51 to 74$)$ \\
\hline DM (\%) & 20.4 & 20.5 & N/A & N/A & 22.6 & 17.2 \\
\hline \multicolumn{7}{|l|}{ Diagnostic category (\%) } \\
\hline Medical & 55.2 & 54.1 & 64.1 & 63.2 & 39.9 & 40.4 \\
\hline Surgical/Trauma & 44.8 & 45.9 & 35.9 & 36.8 & 60.1 & 59.6 \\
\hline Mechanical ventilation (\%) & 45.8 & 35.4 & N/A & N/A & 84.2 & 82.0 \\
\hline ICU LOS & 2.5 (1.1 to 5.7$)$ & 1.5 (0.9 to 3.1$)$ & $3.0(1.8$ to 6.9$)$ & $3.0(1.7$ to 6.3$)$ & $6.0(3.0$ to 13.0$)$ & 6.0 (3.0 to 12.5$)$ \\
\hline APACHE II score & 16 (11 to 22$)$ & $14(10$ to 21$)$ & $19.7(8.2)$ & $19.4(8.3)$ & 15 (11 to 21$)$ & 15 (11 to 21$)$ \\
\hline Mortality (\%) & 19.2 & 14.2 & 27.5 & 26.0 & 17.8 & 22.9 \\
\hline \multicolumn{7}{|l|}{ Glucose control parameters } \\
\hline $\begin{array}{l}\text { Number of BG measurements per } \\
\text { patient }\end{array}$ & 18 (9 to 47$)$ & 15 (8 to 34 ) & 17 (7 to 38$)$ & 29 (12 to 69$)$ & 29 (12 to 74$)$ & 37 (15 to 93$)$ \\
\hline $\begin{array}{l}\text { Number of BG measurements per } \\
\text { day* }^{*}\end{array}$ & 8.7 (5.7 to 10.5$)$ & 9.3 (8.0 to 11.3 ) & 5.1 (3.6 to 7.6$)$ & $9.8(6.8$ to 12.1$)$ & 4.5 (3.2 to 6.7$)$ & 5.6 (3.8 to 9.0$)$ \\
\hline Mean $(\mathrm{mg} / \mathrm{dL})$ & $\begin{array}{l}124.4(112.2 \text { to } \\
140.7)\end{array}$ & $\begin{array}{l}124.0(112.0 \text { to } \\
138.0)\end{array}$ & $\begin{array}{l}127.7(116.4 \text { to } \\
145.0)\end{array}$ & $\begin{array}{l}117.9(107.0 \text { to } \\
137.0)\end{array}$ & $\begin{array}{l}146.3(128.1 \text { to } \\
164.6)\end{array}$ & $\begin{array}{l}118.5(109.3 \text { to } \\
130.3)\end{array}$ \\
\hline CV (\%) & $\begin{array}{l}23.8(16.7 \text { to } \\
32.6)\end{array}$ & $\begin{array}{l}21.0(14.8 \text { to } \\
28.5)\end{array}$ & $\begin{array}{l}26.9(18.8 \text { to } \\
35.2)\end{array}$ & $\begin{array}{l}31.8(23.8 \text { to } \\
40.8)\end{array}$ & $\begin{array}{l}20.7(15.4 \text { to } \\
26.2)\end{array}$ & $\begin{array}{l}26.2(20.3 \text { to } \\
33.1)\end{array}$ \\
\hline $\mathrm{SD}(\mathrm{mg} / \mathrm{dL})$ & $\begin{array}{l}29.5(20.0 \text { to } \\
42.6)\end{array}$ & $\begin{array}{l}25.9(17.7 \text { to } \\
37.5)\end{array}$ & $\begin{array}{l}33.8(23.6 \text { to } \\
49.4)\end{array}$ & $\begin{array}{l}37.1(27.3 \text { to } \\
50.3)\end{array}$ & $\begin{array}{l}29.3(19.9 \text { to } \\
41.7)\end{array}$ & $\begin{array}{l}30.5(22.7 \text { to } \\
42.1)\end{array}$ \\
\hline \multicolumn{7}{|l|}{$\begin{array}{l}\text { Percentage of patients with } \\
\text { hypoglycemia }\end{array}$} \\
\hline$<40 \mathrm{mg} / \mathrm{dL}$ & 6.7 & 2.9 & 8.8 & 18.1 & 2.4 & 9.5 \\
\hline 40 to $54 \mathrm{mg} / \mathrm{dL}$ & 12.6 & 8.4 & 13.6 & 25.2 & 3.9 & 23.1 \\
\hline 55 to $69 \mathrm{mg} / \mathrm{dL}$ & 17.7 & 14.9 & 19.3 & 21.6 & 11.5 & 31.3 \\
\hline$<70 \mathrm{mg} / \mathrm{dL}$ & 37.0 & 26.2 & 41.7 & 64.9 & 17.8 & 63.9 \\
\hline
\end{tabular}

APACHE II, Acute Physiology and Chronic Health Evaluation II disease classification system; BG, blood glucose; CV, coefficient of variation; DM, diabetes mellitus; GL-C, patients in the control arm of the GLUCONTROL trial; GL-IIT, patients in the intervention arm of the GLUCONTROL trial; LOS, length of stay (days); NL-L, Netherlands "loose" cohort; NL-S, Netherlands "strict" cohort; SD, standard deviation; ST, Stamford cohort.

${ }^{*}$ Calculated as (mean number of BG tests/mean ICU LOS). Data expressed as median (interquartile range), or percentages

\section{Sensitivity analysis: association between hypoglycemia and mortality}

Table 3 demonstrates for the entire cohort of 6,240 patients increased relative risk (RR) of mortality for patients with minimum BG $<40,40$ to 54,55 to 69 , 70 to 79 compared to those with a minimum BG of 80 to $109 \mathrm{mg} / \mathrm{dL}$ and Figure 1 stratifies these results by subpopulation. Increasing severity of hypoglycemia was consistently associated with increased risk of mortality. The RR (95\% CI) for mortality associated with hypoglycemia $(B G<70 \mathrm{mg} / \mathrm{dL}$ ) in ST patients was 3.28 (2.78 to 3.87) $P<0.0001$; for NL patients in the "loose" and "strict" cohorts it was 1.53 (1.27 to 1.86) $P$ $<0.0001$ and 1.10 (0.87 to 1.38 ) $P=0.4288$, respectively; and for patients in the GL cohort it was 2.11 (1.62 to 2.74). Figure 2 stratifies this analysis by severity of illness. The association between mortality and mild hypoglycemia is strongly demonstrated for patients with a mild to moderate (APACHE II score < 15 ) and moderately severe (APACHE II score 15 to 24) disease but blunted for those with very severe disease (APACHE II score > 24).

\section{Association between hypoglycemia and mortality, stratified by clinical characteristics of patients}

Table 4 demonstrates that patients with hypoglycemia sustained higher mortality than did those without hypoglycemia, regardless of diagnostic category, diabetic status, ICU LOS and frequency of BG measurements. Table 5 displays the results of multivariate regression analysis of the association between hypoglycemia and mortality for patients with these clinical characteristics, as well as the independent association of mortality associated with $\mathrm{BG}<70 \mathrm{mg} / \mathrm{dL}, \mathrm{BG} 40$ to $69 \mathrm{mg} / \mathrm{dL}$ and $\mathrm{BG}<40 \mathrm{mg} / \mathrm{dL}$.

Mortality associated with number of hypoglycemic events Among 4,906 patients with no or one episode of hypoglycemia, multivariate analysis demonstrated that a single episode of hypoglycemia was independently 
Table 2 Characteristics of patients with hypoglycemia and patients without hypoglycemia

\begin{tabular}{|c|c|c|c|c|c|c|c|c|}
\hline & \multicolumn{2}{|c|}{ ALL } & \multicolumn{2}{|c|}{ ST } & \multicolumn{2}{|c|}{ NL } & \multicolumn{2}{|c|}{$\mathrm{GL}$} \\
\hline & HYPO & NON & HYPO & NON & HYPO & NON & HYPO & NON \\
\hline \multicolumn{9}{|l|}{$\begin{array}{l}\text { Demographics and } \\
\text { outcomes }\end{array}$} \\
\hline Number & 2,309 & 3,921 & 857 & 2,406 & 1,084 & 979 & 372 & 542 \\
\hline Age (years) & 70 (57 to 79$)$ & 66 (52 to 71$)$ & 75 (60 to 83$)$ & 66 (51 to 78$)$ & 69 (58 to 78$)$ & 68 (55 to 78$)$ & 66 (51 to 75$)$ & 65 (52 to 73 ) \\
\hline DM (\%) & 27.3 & 17.5 & 29.6 & 17.2 & N/A & N/A & 22.5 & 18.7 \\
\hline \multicolumn{9}{|l|}{ Diagnostic Category (\%) } \\
\hline Medical & 64.5 & 49.8 & 69.7 & 48.5 & 65.5 & 61.7 & 48.7 & 33.4 \\
\hline Surgical/Trauma & 35.5 & 50.2 & 30.3 & 51.5 & 34.5 & 38.3 & 51.3 & 66.6 \\
\hline Mechanical ventilation & 63.3 & 38.5 & 55.0 & 28.5 & N/A & N/A & 82.6 & 83.5 \\
\hline ICU Length of Stay & $\begin{array}{l}5.0(2.2 \text { to } \\
10.5)\end{array}$ & $\begin{array}{l}1.8(1.0 \text { to } \\
3.3)\end{array}$ & $\begin{array}{l}3.0(1.4 \text { to } \\
7.1)\end{array}$ & $\begin{array}{l}1.2(0.8 \text { to } \\
2.3)\end{array}$ & $\begin{array}{l}5.2(2.6 \text { to } \\
10.3)\end{array}$ & $\begin{array}{l}2.0(1.3 \text { to } \\
3.2)\end{array}$ & 9 (5 to 17$)$ & 5 (3 to 9) \\
\hline APACHE II score & 20 (14 to 26$)$ & $14(10$ to 19$)$ & $21.1(9.3)$ & $14.2(8.1)$ & $21.2(7.8)$ & $17.8(8.4)$ & $18.7(7.5)$ & $15.3(6.5)$ \\
\hline Mortality (\%) & 29.6 & 13.1 & 29.1 & $8.9^{1}$ & 30.1 & 23.2 & 29.6 & 14.0 \\
\hline \multicolumn{9}{|l|}{$\begin{array}{l}\text { Glucose control } \\
\text { parameters }\end{array}$} \\
\hline $\begin{array}{l}\text { Number of BG } \\
\text { measurements per patient }\end{array}$ & 45 (21 to 97 ) & 11 (7 to 24$)$ & 35 (16 to 90 ) & 11 (7 to 23) & 47 (24 to 92 ) & 9 (5 to 18$)$ & $\begin{array}{l}69(29 \text { to } \\
136)\end{array}$ & 20 (10 to 48 ) \\
\hline $\begin{array}{l}\text { Number of BG } \\
\text { measurements per day* }\end{array}$ & $\begin{array}{l}9.5(7.2 \text { to } \\
11.9)\end{array}$ & $\begin{array}{l}8.0(5.0 \text { to } \\
10.0)\end{array}$ & 11.7 & 9.2 & 9.0 & 4.5 & 7.7 & 4.0 \\
\hline Mean $(\mathrm{mg} / \mathrm{dL})^{\wedge}$ & $\begin{array}{l}118.3(108.1 \\
\text { to } 132.5)\end{array}$ & $\begin{array}{l}128.1(115.3 \\
\text { to } 144.4)\end{array}$ & $\begin{array}{l}120.3(108.4 \\
\text { to } 133.9)\end{array}$ & $\begin{array}{l}125.4(113.4 \\
\text { to } 138.6)^{1}\end{array}$ & $\begin{array}{l}116.9(107.5 \\
\text { to } 129.9)\end{array}$ & $\begin{array}{l}131.9(119.2 \\
\text { to } 152.6)\end{array}$ & $\begin{array}{l}119.1(109.0 \\
\text { to } 136.3)\end{array}$ & $\begin{array}{l}137.5(121.0 \\
\text { to } 158.4)\end{array}$ \\
\hline CV (\%) & $\begin{array}{l}31.6(25.0 \text { to } \\
40.0)\end{array}$ & $\begin{array}{l}19.2(13.7 \text { to } \\
26.1)\end{array}$ & $\begin{array}{l}29.3(23.1 \text { to } \\
38.2)\end{array}$ & $\begin{array}{l}18.4(13.0 \text { to } \\
24.6)\end{array}$ & $\begin{array}{l}34.2(23.0 \text { to } \\
42.4)\end{array}$ & $\begin{array}{l}21.7(15.0 \text { to } \\
30.2)\end{array}$ & $\begin{array}{l}27.7(22.6 \text { to } \\
35.5)\end{array}$ & $\begin{array}{l}20.2(15.2 \text { to } \\
25.8)\end{array}$ \\
\hline SD (mg/dL) & $\begin{array}{l}24.8(16.7 \text { to } \\
36.0)\end{array}$ & $\begin{array}{l}37.6(28.9 \text { to } \\
50.4)\end{array}$ & $\begin{array}{l}34.9(26.2 \text { to } \\
48.8)\end{array}$ & $\begin{array}{l}22.7(15.4 \text { to } \\
32.5)\end{array}$ & $\begin{array}{l}40.2(31.2 \text { to } \\
54.1)\end{array}$ & $\begin{array}{l}28.1 \text { (18.8 to } \\
44.5)\end{array}$ & $\begin{array}{l}34.8(25.7 \text { to } \\
46.6)\end{array}$ & $\begin{array}{l}27.5(19.1 \text { to } \\
38.8)\end{array}$ \\
\hline
\end{tabular}

*Calculated as (mean number of BG tests/mean ICU LOS)

$\wedge$ These values represent the median (IQR) of the individual patients' mean BG

Data expressed as median (interquartile range), or percentages

APACHE II, Acute Physiology and Chronic Health Evaluation II disease classification system; BG, blood glucose; CV, coefficient of variation; DM, diabetes mellitus; GL, GLUCONTROL cohort; HYPO, hypoglycemia (BG < 70 mg/dL); LOS, length of stay (days); NL, Netherlands cohort; NON, no hypoglycemia; SD, standard deviation; ST, Stamford cohort.

associated with increased risk of mortality: OR (95\% CI) 1.31 (1.06 to 1.62 ) $P=0.0121$. Among 5,535 patients with up to three episodes of hypoglycemia, multivariate analysis demonstrated that hypoglycemia was independently associated with increased risk of mortality: OR

Table 3 Relative risk of mortality: comparison to patients with a minimum BG of 80 to $110 \mathrm{mg} / \mathrm{dL}$

\begin{tabular}{llll}
\hline $\begin{array}{l}\text { Minimum BG (mg/ } \\
\text { dL) }\end{array}$ & $\begin{array}{l}\text { Number of } \\
\text { patients }\end{array}$ & RR $(\mathbf{9 5 \%}$ Cl) & P-value \\
\hline$<40$ & 421 & $\begin{array}{l}3.55(3.02 \text { to } \\
4.17)\end{array}$ & $<$ \\
& & $2.70(2.31$ to & $<$ \\
40 to 55 & 789 & $3.14)$ & 0.0001 \\
& & $2.18(1.87$ to & $<$ \\
55 to 70 & 1,103 & $2.53)$ & 0.0001 \\
& & $1.43(1.18$ to & 0.0002 \\
70 to 80 & 854 & $1.73)$ & \\
& & Reference & \\
80 to 110 & 2,383 & $1.35(1.10$ to & 0.0046 \\
$\geq 110$ & 690 & $1.66)$ & \\
\hline
\end{tabular}

BG, blood glucose; $\mathrm{Cl}$, confidence interval; $\mathrm{RR}$, relative risk
(95\% CI) 1.35 (1.14 to 1.61$) P=0.0007$ Among the entire cohort of 6,240 patients, patients with four or more episodes of hypoglycemia had OR for mortality: 1.49 (1.20 to 1.83 ) $P=0.0002$ (compared to patients without hypoglycemia).

\section{Association between hypoglycemia and mortality, stratified by mean BG concentration and coefficient of variation}

Figure 3 displays mortality stratified by bands of mean BG during ICU stay: < $80 \mathrm{mg} / \mathrm{dL}, 80$ to $110 \mathrm{mg} / \mathrm{dL}, 110$ to $140 \mathrm{mg} / \mathrm{dL}, 140$ to $180 \mathrm{mg} / \mathrm{dL}$ and $\geq 180 \mathrm{mg} / \mathrm{dL}$, in patients with and without hypoglycemia. Among patients with mean BG 80 to $110 \mathrm{mg} / \mathrm{dL}$, hypoglycemia was associated with a 4-fold increase in mortality; the "hypoglycemia penalty" was 2.8-fold for patients with mean BG 110 to 140 and 2-fold for patients with a mean BG of 140 to $180 \mathrm{mg} / \mathrm{dL}$. Among patients with $\mathrm{CV}<15 \%, 15$ to $30 \%$ and $\geq 30 \%$ mortality was higher in patients with hypoglycemia than in patients without hypoglycemia: $13.3 \%$ vs. $6.9 \%(P=0.3216), 26.4 \%$ vs. 


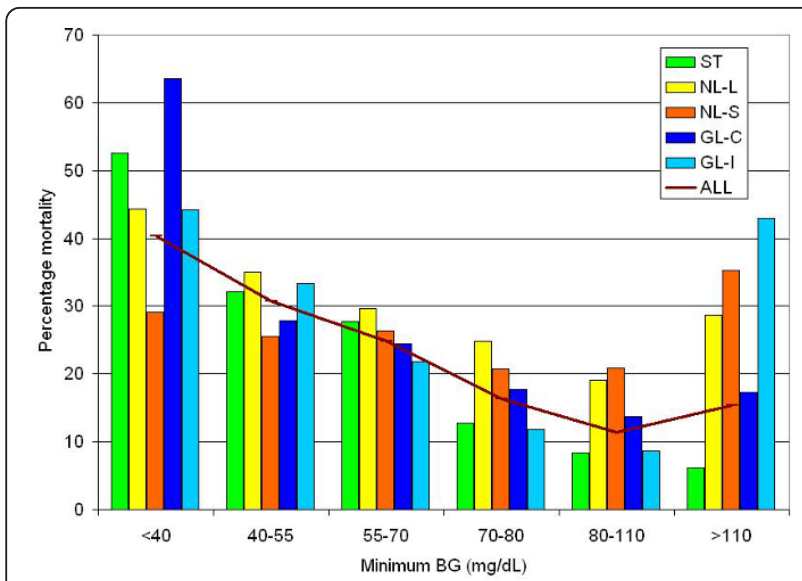

Figure 1 Relationship between minimum BG during ICU stay and mortality, stratified by subpopulation. $\mathrm{BG}$, blood glucose; GL-C, GLUCONTROL-control arm; GL-I, GLUCONTROL-intensive arm; NL-L, Netherlands-"loose"; NL-S, Netherlands-"strict"; ST, Stamford.

$14.3 \%(P<0.0001)$ and $32.5 \%$ vs. $21.2 \%(P<0.0001)$. Finally, Figure 4 presents unadjusted mortality rates for patients with differing mean blood glucose, differing coefficient of variation of blood glucose and presence or absence of hypoglycemia. The highest crude mortality rates were observed in patients with higher mean blood glucose and coefficient of variation of blood glucose who experience hypoglycemia. Patients with hypoglycemia, high glycemic variability and high mean BG sustained 7.5-fold higher mortality than did those with low glycemic variability, low mean BG and no hypoglycemia.

\section{Discussion}

This study examined the relationship between hypoglycemia occurring during ICU stay and hospital mortality in

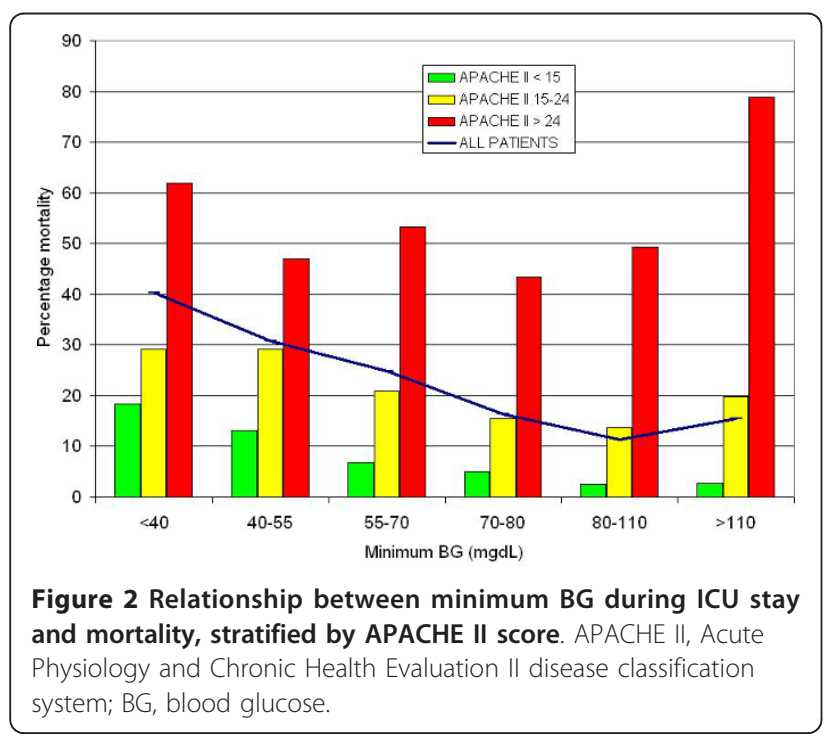

Table 4 Mortality percentages of patients with hypoglycemia and without hypoglycemia, stratified by different clinical characteristics

\begin{tabular}{llll}
\hline Characteristic & Hypoglycemia & No hypoglycemia & $P$-value \\
\hline Diagnostic category & & & \\
Medical & 33.9 & 19.2 & $<0.0001$ \\
Surgical/trauma & 21.8 & 7.1 & $<0.0001$ \\
Diabetes & 27.2 & 10.5 & $<0.0001$ \\
No diabetes & 30.0 & 9.7 & $<0.0001$ \\
ICU LOS & & & $<0.0001$ \\
$<\mathbf{3}$ days & 28.3 & 11.4 & $<0.0001$ \\
$\mathbf{3}$ to 7 days & 29.0 & 14.6 & 0.0024 \\
$>\mathbf{7}$ days & 30.6 & 22.2 & $<0.0001$ \\
Frequency of BG tests & & & $<0.0001$ \\
$<\mathbf{6 / d a y}$ & 25.2 & 14.4 & $<0.0001$ \\
$\mathbf{6}$ to 9/day & 31.3 & 12.1 & \\
$>$ 9/day & 29.9 & 13.0 &
\end{tabular}

BG, blood glucose; LOS, length of stay

three cohorts of patients. The salient finding is that even a single episode of mild hypoglycemia, defined as BG < $70 \mathrm{mg} / \mathrm{dL}$, was associated with increased risk of mortality. A major strength of this investigation includes the nature of the aggregated patient cohort, involving patients from different countries, with varying severities of illness and ICU LOS, treated in ICU's using different glycemic targets, measurement technologies and glycemic management protocols. Notably, the association between hypoglycemia and mortality was different among cohorts with different strategies of glucose control. The highest relative risk for mortality was seen in the cohort with the lowest rates of hypoglycemia while the lowest risk for mortality was seen in the cohort in whom short episodes of mild hypoglycemia were accepted as part of the guideline for IIT. The association between hypoglycemia and mortality was independent of diagnostic category and diabetic status and was seen predominantly in patients with mild to moderate and moderate to severe disease on presentation to the ICU, reflected by APACHE II scores 0 to 14 and 15 to 24 respectively. Finally, the association of hypoglycemia with mortality was cumulative to the associations of hyperglycemia and increased glycemic variability with mortality [20].

This investigation expands upon earlier work studying the association of hypoglycemia with mortality in the critically ill. It is notable that the investigators of the first prospective randomized controlled trial of IIT [4] and a subsequent confirmatory before and after investigation [5] stated that severe hypoglycemia ( $\mathrm{SH}$, defined as $B G<40 \mathrm{mg} / \mathrm{dL}$ ) did not have an independent effect on mortality. However, a subsequent pooled analysis of the two Leuven trials [13] determined that the OR for mortality associated with a single episode of $\mathrm{SH}$ was $3.23(2.25$ to 4.64$)(P<0.0001)$. This compares to the 
Table 5 Multivariate analysis

\begin{tabular}{|c|c|c|c|c|c|c|}
\hline & \multicolumn{2}{|c|}{ Minimum BG $<40 \mathrm{mg} / \mathrm{dL}$} & \multicolumn{2}{|c|}{ Minimum BG 40 to $69 \mathrm{mg} / \mathrm{dL}$} & \multicolumn{2}{|c|}{ Minimum BG $<70 \mathrm{mg} / \mathrm{dL}$} \\
\hline & OR $(95 \% \mathrm{Cl})$ & $P$-value & OR $(95 \% \mathrm{Cl})$ & $P$-value & OR $(95 \% \mathrm{Cl})$ & $P$-value \\
\hline $\begin{array}{l}\text { Entire cohort }^{1} \\
(n=6,240)\end{array}$ & 1.49 (1.14 to 1.94$)$ & 0.0031 & 1.17 (0.99 to 1.38$)$ & 0.0557 & $1.47(1.22$ to 1.78$)$ & $<0.0001$ \\
\hline $\begin{array}{l}\text { No diabetes }{ }^{2} \\
(n=3,326)\end{array}$ & $2.42(1.42$ to 4.10$)$ & 0.0011 & $1.33(1.02$ to 1.75$)$ & 0.0370 & 1.65 (1.24 to 2.19$)$ & 0.0005 \\
\hline $\begin{array}{l}\text { Diabetes }^{2} \\
(n=850)\end{array}$ & 2.37 (1.12 to 5.01$)$ & 0.0236 & 1.58 (0.99 to 2.52$)$ & 0.0549 & $2.48(1.48$ to 4.15$)$ & 0.0005 \\
\hline $\begin{array}{l}\text { Medical admission }{ }^{1} \\
(n=3,441)\end{array}$ & 1.44 (1.02 to 2.03$)$ & 0.0395 & $1.06(0.85$ to 1.32$)$ & 0.6165 & $1.28(1.00$ to 1.63$)$ & 0.0472 \\
\hline Surgical/trauma admission ${ }^{1}(n=2,787)$ & 1.59 (1.05 to 2.40$)$ & 0.0283 & 1.34 (1.04 to 1.72$)$ & 0.0220 & 1.77 (1.34 to 2.34$)$ & $<0.0001$ \\
\hline
\end{tabular}

Multivariate model includes age; modified APACHE II score (age component deleted); CV; ICU LOS; mean BG; medical vs. surgical/trauma admission; mechanical ventilation (as indicated)

${ }^{1}$ Does not include mechanical ventilation in the model

2 Excludes NL patients (for whom MV and diabetes data not available); includes MV in the model

APACHE II, Acute Physiology and Chronic Health Evaluation II disease classification system; BG, blood glucose; CV, coefficient of variation; LOS, length of stay; NL, Netherlands cohort; OR, odds ratio

OR for mortality associated with a single episode of $\mathrm{SH}$ reported from two large observational series, 2.28 (1.41 to 3.70$)$ in a cohort of 5,365 mixed medical-surgical patients from a single ICU in the USA [10] and $2.6(2.1$ to 3.2 ) in a cohort of 66,184 patients admitted to 24 Australian ICUs [11]. Recently, Egi et al. investigated the relationship between milder degrees of hypoglycemia and mortality in a population of 4,946 patients admitted to two Australian ICUs with glycemic targets of 108 to $180 \mathrm{mg} / \mathrm{dL}$ [14]. Multivariate analysis revealed that patients with a minimum BG of 54 to $63 \mathrm{mg} / \mathrm{dL}$ had significantly higher risk of mortality than did those with a minimum BG of 72 to $81 \mathrm{mg} / \mathrm{dL}$, OR 1.93 (1.27 to 2.95) $(P=0.002)$ - as well as significantly higher risk of infection - OR 2.16 (1.17 to 3.99) $(P=0.01)$. The findings of the current investigation corroborate the

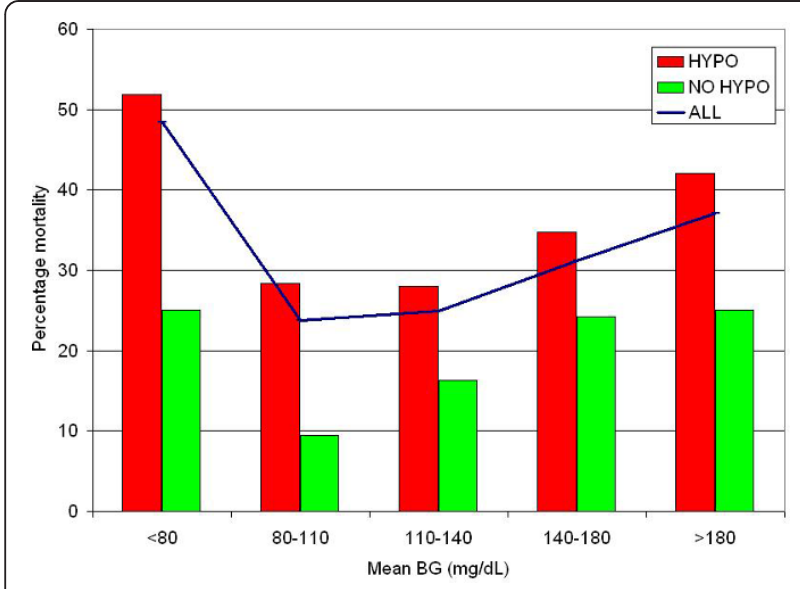

Figure 3 Relationship of mean blood glucose-mortality, stratified by presence or absence of hypoglycemia. There were 32 patients with mean $\mathrm{BG}<80 \mathrm{mg} / \mathrm{dL}$, 28 with hypoglycemia (51.9\% mortality) and 1 without hypoglycemia (25\% mortality). BG, blood glucose; HYPO; hypoglycemia BG $<70$ mg/dL. association of mild hypoglycemia with increased risk of mortality in critically ill patients demonstrated in the study by Egi and coworkers.

The strengths of the current investigation include, in part, the diverse nature of the different patient populations and the breadth of the dataset used for analysis. This international collaboration comprises large, well described observational cohorts from one ICU in the USA and three ICUs in The Netherlands, as well as the 21 ICUs participating in one of the major randomized controlled trials of intensive insulin therapy [8], increasing the generalizability of its findings. We acknowledge several limitations in the dataset used for this analysis. The use of bedside glucometers in a percentage of patients in all three cohorts and capillary fingerstick blood in a percentage of the patients in the ST and GL cohorts is a potential limitation as this technology has

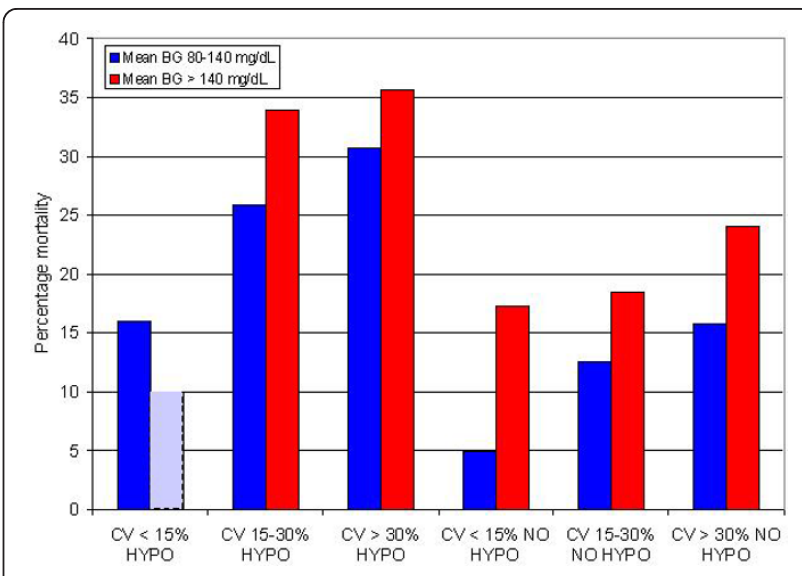

Figure 4 Association of mortality with disturbances in the three domains of glycemic control. There were no patients with Mean BG > $140 \mathrm{mg} / \mathrm{dL}$ and CV < 15\%. BG, blood glucose; CV, coefficient of variation; HYPO, hypoglycemia BG $<70 \mathrm{mg} / \mathrm{dL}$. 
been associated with analytic inaccuracies in critically ill populations [21-23]. Nevertheless, this measurement technology reflects "real world" practices. Moreover, any "scatter" of the data induced by a measurement technology less accurate than a central laboratory analyzer would serve to "dampen" the signal of the association of hypoglycemia and mortality in this population. It is notable that Egi et al. [14], in a study that solely used arterial blood gas analyzers for glucose measurement, identified a threshold for the association between hypoglycemia and mortality that was similar to that seen in our investigation. While the manual and intermittent nature of monitoring BG in all three cohorts likely led to an incomplete accounting of all the hypoglycemia that occurred in the patients studied, due to the nursing time required for compliance with even hourly BG [24], it is likely that the size of the combined cohort was sufficient to examine the endpoints chosen. The absence of information about nutritional support, insulin treatment and glycemic control after ICU stay are additional limitations in the dataset used for this analysis. Finally, there are no data available to evaluate the relationship between the timing of the hypoglycemic events and death, or the specific causes of death for patients in the three cohorts.

There are some possible links between hypoglycemia and worsened outcome or complicated course of critical illness [25]. First, the physiological mechanisms triggered by hypoglycemia are commonly impaired during critical illness. These include the inhibition of insulin release, typically occurring when BG is lower than $80 \mathrm{mg} / \mathrm{dl}$ [26]; an increased release of glucagon, epinephrine and growth hormone when BG is lower than $65 \mathrm{mg} / \mathrm{dl}$ and increased release of cortisol when BG is lower than $55 \mathrm{mg} / \mathrm{dl}$ [27]. During critical illness, exogenous insulin is infused and the levels of glucagon, epinephrine, cortisol and growth hormone are typically already elevated. Second, large swings in BG, as observed when hypoglycemia is aggressively treated with a large amount of intravenous glucose, may be associated with cellular damages [28]. Unfortunately, data detailing the amount of glucose given to treat hypoglycemia and the neurological status of the patients in our three cohorts were not available. Third, the detrimental effects of hypoglycemia are well documented in the brain. Indeed, glucose is the preferential energetic substrate in the brain. The absence of cerebral stores of glucose and the diffusive character of transport imply that the glucose concentration in neurons and glial cells is entirely determined by BG [29]. Therefore, brain injured patients are at higher risk of hypoglycemia-related damage; conversely, hypoglycemia also induces brain dysfunction even in patients without prior cerebral compromise.
This study has a number of important clinical implications. While earlier investigations detailed the independent association of severe hypoglycemia with increased risk of mortality in critically ill patients [10-13], the data from the current work suggest strongly that milder degrees of hypoglycemia are also associated with harm. Moreover, mild hypoglycemia was associated with increased risk of mortality among patients admitted with medical as well as surgical diagnoses, among patients with diabetes and those without, and among patients with mild-moderate disease and moderatesevere disease, as reflected by APACHE II score (but not among patients with APACHE II scores $\geq 25$, in whom the severity of illness may have overwhelmed the independent impact of hypoglycemia).

Interestingly, the signal for harm varied in the three cohorts. The relative risk of mortality associated with hypoglycemia was strongest for patients in the ST cohort, intermediate for patients in the GL cohort, and smallest for those in the NL cohort, inversely related to the prevalence of hypoglycemia in the three populations of patients. One explanation of this finding is the different distribution of severity of illness in the cohorts, as reflected by APACHE II scores. More patients in the NL cohort had APACHE II scores $\geq 25$ than did patients in the ST and GL cohort. Figure 2 demonstrates that there was no association between hypoglycemia and mortality among patients in these groups; perhaps the severity of illness lowered the sensitivity of the association of hypoglycemia with increased risk of mortality. In addition, patients in the "strict" IIT cohort in the NL, with a glycemic target of euglycemia, were treated using a guideline that allowed brief excursions into the 60 to $80 \mathrm{mg} / \mathrm{dL}$, resulting in a higher rate of hypoglycemia than was seen in the other cohorts. The IIT guideline used for the NL cohort also allowed patients to gradually recover from hypoglycemia, avoiding the administration of quantities of dextrose that might lead to sharp spikes in glucose levels and, consequently, excessive glucose variability, recently identified as having an independent association with increased risk of mortality in critically ill patients [13,30-32]. This practice could be a second reason that the sensitivity of the association between hypoglycemia and mortality was lowered in the NL-S cohort. Additional differences between the NL-L and NL-S cohorts included the exclusive use of arterial blood for measurement of BG in the IIT group, and significantly higher glycemic variability, as reflected by CV and SD, in the NL-S cohort. Thus, any future evaluations of the association of hypoglycemia with mortality in critically ill populations may benefit from an analysis of the severity of illness, the prevalence and practice of treating hypoglycemia, as well as the glycemic management protocol employed. 
Our investigation contrasts with the finding in a recent study of a U-shaped curve relating mean BG to mortality in critically ill patients [33]. The lowest mortality was associated with a mean BG of 80 to $110 \mathrm{mg} /$ $\mathrm{dL}$ and 110 to $140 \mathrm{mg} / \mathrm{dL}$ during ICU stay. Patients with hypoglycemia in these two bands of mean BG had very similar mortality, but 4.3 and 2.6 times the rate of mortality as those without hypoglycemia, respectively. Higher bands of mean BG were associated with even higher rates of mortality, both for patients with and without hypoglycemia. These observations are parallel to some of the key findings of the major interventional trials of intensive insulin therapy. The sharply higher mortality observed in patients with a mean BG of 80 to $110 \mathrm{mg} / \mathrm{dL}$ and hypoglycemia compared to those with a mean BG of 80 to $110 \mathrm{mg} / \mathrm{dL}$ and no hypoglycemia highlights the different outcomes of Leuven 1 [4] and 2 [6]. In the medical ICU trial [6] the markedly higher rate of severe hypoglycemia in patients attenuated the beneficial impact of intensive insulin therapy observed in the surgical ICU trial [4]. Moreover, while in both trials, the "conventional" arm had a glycemic target range of 180 to $200 \mathrm{mg} / \mathrm{dL}$, our investigation demonstrated that patients with mean BG $\geq 180 \mathrm{mg} / \mathrm{dL}$ and no hypoglycemia sustained even higher mortality than did those with mean BG of 80 to $110 \mathrm{mg} / \mathrm{dL}$ with hypoglycemia. Finally, in our study, patients with hypoglycemia and a mean BG of 80 to $110 \mathrm{mg} / \mathrm{dL}$ sustained higher mortality than did those without hypoglycemia and a mean BG of 140 to $180 \mathrm{mg} / \mathrm{dL}$, analogous to the conclusions of the NICE to SUGAR trial, in which the 13.6fold higher rate of severe hypoglycemia in patients treated in the interventional arm compared to those in the control arm was associated with higher mortality [9].

\section{Conclusions}

This investigation supports and extends the findings of other recent studies of the impact of hypoglycemia in the critically ill and suggests that mild hypoglycemia, defined as $\mathrm{BG}<70 \mathrm{mg} / \mathrm{dL}$, is independently associated with increased risk of mortality. Of course, it would certainly be unethical and, therefore, impossible to perform a randomized trial targeting hypoglycemia in a group of critically ill patients. However, while causality cannot be proven by these data - they must be considered hypothesis generating - the findings of this investigation suggest that critical care teams should attempt to avoid even modest degrees of hypoglycemia in their patients.

\section{Key messages}

- Mild hypoglycemia, defined as BG $<70 \mathrm{mg} / \mathrm{dL}$, was associated with increased risk of mortality in a diverse and heterogeneous group of three patient cohorts.
- The relative risk of mortality associated with hypoglycemia differed among the cohorts.

- The association of mild hypoglycemia with mortality was observed in medical and surgical patients, in diabetics and non to diabetics and it occurred independently of mean BG during ICU stay.

- The association of mild hypoglycemia with mortality was strongest in patients with mild to moderate and moderate to severe disease upon presentation to the ICU, reflected by APACHE II scores $<15$ and 15 to 24, respectively, and was weaker in patients with APACHE II scores $\geq 25$.

- Efforts to reduce the occurrence of mild hypoglycemia in critically ill patients may reduce mortality.

\section{Additional material}

Additional file 1: Stamford Hospital glycemic management protocol. Additional file 2: GLUCONTROL trial glycemic management protocols.

\section{Abbreviations}

APACHE II: Acute Physiology and Chronic Health Evaluation II disease classification system; BG: blood glucose; Cl: confidence interval; CV: coefficient of variation; DM: diabetes mellitus; GL: GLUCONTROL cohort; GLC: GLUCONTROL-control arm; GL-C: patients in the control arm of the GLUCONTROL trial; GL-I: GLUCONTROL-intensive arm; GL-IIT: patients in the intervention arm of the GLUCONTROL trial; HYPO: hypoglycemia (BG < 70 $\mathrm{mg} / \mathrm{dL}$ ); LOS: length of stay; NL: Netherlands cohort; NL-L: Netherlands "loose" cohort; NL-S: Netherlands "strict" cohort; NON: no hypoglycemia; RR: relative risk; SD: standard deviation; ST: Stamford cohort.

\section{Author details}

${ }^{1}$ Division of Critical Care, Stamford Hospital, Columbia University College of Physicians and Surgeons, 190 West Broad Street, Stamford, CT 06902, USA. ${ }^{2}$ Department of Intensive Care, Academic Medical Center, University of Amsterdam, Meibergdreef 9, 1105 AZ Amsterdam, The Netherlands. ${ }^{3}$ Laboratory of Experimental Intensive Care and Anesthesiology, Academic Medical Center, University of Amsterdam, Meibergdreef 9, 1105 AZ

Amsterdam, The Netherlands. ${ }^{4}$ Department of Intensive Care, Gelre Hospitals, Lukas, Albert Schweitzerlaan 31, 7334 DZ Apeldoorn, The Netherlands. ${ }^{5}$ Department of Intensive Care, Tergooi Hospitals, Blaricum, Rijksstraatweg 1, 1261 AN Blaricum, The Netherlands. 'Department of Intensive Care Medicine, Medical Center Haaglanden, Lijnbaan 32, The Hague, 2512 VA Den Haag, The Netherlands. ${ }^{7}$ Department of Emergency Medicine, Erasme University Hospital, Route de Lennik 808, 1070 Bruxelles, Belgium. ${ }^{8}$ Department of Intensive Care, Erasme University Hospital, Route de Lennik 808, 1070 Bruxelles, Belgium.

\section{Authors' contributions}

JSK wrote the initial and subsequent drafts of the manuscript and performed statistical analysis. MJS and JCP reviewed all drafts of the manuscript and assisted with revisions. PES, REH, FvBH, JPvdS and CM participated in data acquisition and reviewed the final draft of the manuscript. All authors have read and approved the final manuscript for publication.

\section{Competing interests}

James S. Krinsley MD has performed consulting work for Medtronic Inc., Edwards Life Sciences, Baxter, Roche Diagnostics, and Optiscan Biomedical and has received speaker's fees from Edwards Life Sciences, Roche Diagnostics and Sanofi to Aventis. Marcus J. Schultz MD, PhD has performed consulting work for Medtronic Inc., Roche Diagnostics and Optiscan 
Biomedical, and has received research support from Optiscan Biomedical. Jean-Charles Preiser MD, PhD has performed consulting work for Medtronic Inc., Edwards Life Sciences and Optiscan Biomedical. All other authors have no competing interests.

Received: 9 February 2011 Revised: 17 June 2011

Accepted: 25 July 2011 Published: 25 July 2011

\section{References}

1. Dungan S, Braithwaite S, Preiser JC: Stress hyperglycemia. Lancet 2009, 373:1798-1807.

2. Krinsley JS: Association between hyperglycemia and increased hospital mortality in a heterogeneous population of critically ill patients. Mayo Clin Proc 2003, 78:1471-1478.

3. Falciglia M, Freyberg R, Almenoff PL, D'Allesio D, Rener M: Hyperglycemiarelated mortality in critically ill patients varies with admission diagnosis. Crit Care Med 2009, 37:3001-3009.

4. Van den Berghe G, Wouters P, Weekers F, Verwaest C, Bruyninckx F, Schetz M, Vlasslaers D, Ferdinande P, Lauwers P, Bouillon R: Intensive insulin therapy in the critically ill patients. N Engl J Med 2001, 345:1359-1367.

5. Krinsley JS: The effect of an intensive glucose management protocol on the mortality of critically ill adult patients. Mayo Clin Proc 2004, 79:992-1000.

6. Van den Berghe G, Wilmer A, Hermans G, Meersseman W, Wouters PF, Milants I, Van Wijngaerden E, Bobbaers H, Bouillon R: Intensive insulin therapy in the medical ICU. N Engl J Med 2006, 354:449-461.

7. Brunkhorst FM, Engel C, Bloos F, Meier-Hellmann A, Ragaller M, Weiler N, Moerer O, GruendLing M, Oppert M, Grond S, Olthoff D, Jaschinski U, John S, Rossaint R, Welte T, Schaefer M, Kern P, Kuhnt E, Kiehntopf M, Hartog C, Natanson C, Loeffler M, Reinhart K: Intensive insulin therapy and pentastarch resuscitation in severe sepsis. N Eng J Med 2008, 358:125-139.

8. Preiser JC, Devos P, Ruiz-Santana S, Melot C, Annane D, Groeneveld J, lapichino G, Leverve X, Nitenberg G, Singer P, Wernerman J, Joannidis M, Stecher A, Chiolero R: A prospective randomized multi-centre controlled trial on tight glucose control by intensive insulin therapy in adult intensive care units: the GLUCONTROL study. Intensive Care Med 2009, 35:1738-1748.

9. NICE-SUGAR Study Investigators, Finfer S, Chittock DR, Su SY, Blair D, Foster D, Dhingra V, Bellomo R, Cook D, Dodek P, Henderson WR, Hébert PC, Heritier S, Heyland DK, McArthur C, McDonald E, Mitchell I, Myburgh JA, Norton R, Potter J, Robinson BG, Ronco JJ: Intensive versus conventional glucose control in critically ill patients. N Eng J Med 2009, 360:1283-1297.

10. Krinsley JS, Grover A: Severe hypoglycemia in critically ill patients: risk factors and outcomes. Crit Care Med 2007, 35:2262-2267.

11. Bagshaw S, Egi M, George C, Bellomo R: Early blood glucose control and mortality in critically ill patients in Australia. Crit Care Med 2009, 37:463-470.

12. Hermanides J, Bosman RJ, Vriesendorp TM: Hypoglycemia is associated with intensive care unit mortality. Crit Care Med 2010, 38:1430-1434.

13. Meyfroidt G, Keenan DM, Wang X, Wouters P, Veldhuis J, Van den Berghe G: Dynamic characteristics of blood glucose time series during the course of critical illness: effects of intensive insulin therapy and relative association with mortality. Crit Care Med 2010, 38:1021-1029.

14. Egi M, Bellomo R, Stachowski E, French C, Hart G, Taori G, Hegarty C, Bailey M: Hypoglycemia and outcome in critical illness. Mayo Clin Proc 2010, 85:217-224.

15. American Diabetes Association: Standards of care in diabetes. Diabetes Care 2010, 33:S11-S61.

16. Krinsley JS: Glycemic control, diabetic status and mortality in a heterogeneous population of critically ill patients before and during the era of tight glycemic control. Semin Thorac Cardiovasc Surg 2006, 18:317-325.

17. Dellinger RP, Carlet JM, Masur H, Gerlach H, Calandra T, Cohen J, GeaBenacloche J, Keh D, Marshall J, Parker M: Surviving Sepsis Campaign guidelines for management of severe sepsis and septic shock. Intensive Care Med 2004, 30:536-555.

18. Schultz MJ, Harmsen RE, Spronk PE: Strict or loose glycemic control in critically ill patients to implementing best available evidence from randomized controlled trials. Crit Care 2010, 14:223.
19. Arts D, de Keizer N, Scheffer GJ, de Jonge E: Quality of data collected for severity of illness scores in the Dutch National Intensive Care Evaluation (NICE) registry. Intensive Care Med 2002, 28:656-659.

20. Mackenzie I, Whitehouse T, Nightingale P: The metrics of glycaemic control in critical care. Intensive Care Med 2011, 37:435-443.

21. Finkielman J, Oyen $L$, Afessa B: Agreement between bedside and plasma glucose measurement in the ICU setting. Chest 2005, 127:1749-1751.

22. Desachy A, Vuagnat AC, Ghazali AD, Baudin OT, Longuet OH, Calvat SN, Gissot V: Accuracy of bedside glucometry in critically ill patients: Influence of clinical characteristics and perfusion index. Mayo Clin Proc 2008, 83:400-405.

23. Kanji S, Buffie J, Hutton B, Bunting PS, Singh A, McDonald K, Fergusson D, McIntyre LA, Hebert PC: Reliability of point-of-care testing for glucose measurement in critically ill patients. Crit Care Med 2005, 33:2778-2785.

24. Aragon D: Evaluation of nursing work effort and perceptions about blood glucose testing in tight glycemic control. Am J Crit Care 2006, 15:370-377.

25. Lacherade JC, Jacqueminet S, Preiser JC: An overview of hypoglycemia in the critically ill. J Diabetes Sci Technol 2009, 3:1242-1249.

26. Cryer PE, Davis SN, Shamoon H: Hypoglycemia in diabetes. Diabetes Care 2003, 26:1902-1912.

27. Cryer PE: Hypoglycemia: functional brain failure, and brain death. J Clin Invest 2007, 117:868-870.

28. Ceriello A: New insights on oxidative stress and diabetic complications may lead to a "causal" antioxidant therapy. Diabetes Care 2003, 26:1589-1596.

29. Oddo M, Schmidt JM, Carrerra E, Badjatia N, Connolly E, Presciutti M, Ostapkovich N, Levine J, Roux P, Mayer S: Impact of tight glycemic control on cerebral glucose metabolism after severe brain injury: A microdialysis study. Crit Care Med 2008, 36:3233-3238.

30. Egi M, Bellomo R, Stachowski E, French C, Hart G: Variability of blood glucose concentration and short-term mortality in critically ill patients. Anesthesiology 2006, 105:244-252.

31. Krinsley JS: Glycemic variability: a strong, independent risk factor for mortality in critically ill patients. Crit Care Med 2008, 36:3008-3013.

32. Hermanides J, Vriesendorp T, Bosman R, Zandstra D, Hoekstra J, DeVries J: Glucose variability is associated with intensive care unit mortality. Crit Care Med 2010, 38:838-842.

33. Siegelaar SE, Hermanides J, Oudemans-van Straaten HM, van der Voort PHJ, Bosman RJ, Zandstra DF, DeVries JH: Mean glucose during ICU admission is related-mortality by a U-shaped curve in surgical and medical patients: a retrospective cohort study. Crit Care 2010, 14:R224.

doi:10.1186/cc10322

Cite this article as: Krinsley et al:: Mild hypoglycemia is independently associated with increased mortality in the critically ill. Critical Care 2011 15:R173.

\section{Submit your next manuscript to BioMed Central and take full advantage of:}

- Convenient online submission

- Thorough peer review

- No space constraints or color figure charges

- Immediate publication on acceptance

- Inclusion in PubMed, CAS, Scopus and Google Scholar

- Research which is freely available for redistribution

Submit your manuscript at www.biomedcentral.com/submit
Ciomed Central 